JOURNAL OF THEORETICAL

AND APPLIED MECHANICS

55, 1, pp. 29-39, Warsaw 2017

DOI: $10.15632 /$ jtam-pl.55.1.29

\title{
THE APPLICATION OF SELF-OSCILLATION IN WIRE GAUGES
}

\author{
Grzegorz Cieplok, Łukasz KopiJ \\ AGH University of Science and Technology, Kraków, Poland \\ e-mail: cieplok@agh.edu.pl; lkopij@agh.edu.pl
}

\begin{abstract}
In the presented paper, an original solution to the system maintaining vibrations of a wire transducer, which has been based upon the phenomenon of self-oscillation, is described. The vibrations generated in this manner do not fade in time, are resistant to disturbances and allow measurement of the signal rapidly changing in time. The dynamic equations and numerical simulations of motion of the wire interconnected with the van der Pol oscillator are presented. Based on the conceptual model, a laboratory stand has been built, which served to verify properties of the presented solution and possibilities of useful applications.
\end{abstract}

Keywords: self-oscillation, wire transducer

\section{Introduction}

The measurement technique using wire transducers has been known since 1931, when French engineer André Coyne became the first scientist to patent a sensor working on the principle of vibrating wires (Bordes, 2011). In the 1930s, wire sensors became very popular in the technical health monitoring of water dams. The first measurement system was applied to the dam across the Truyere River in Bomme, France (Bordes, 2011). This method was also applied to control technical health of bridges (Bar et al., 2012; Gastineau et al., 2009; Wenzel, 2009), tunnels, large-scale halls, and is currently also being applied in stadiums and transport infrastructure.

Wire measurement is currently an important branch of surveying, being applied in the measurement of displacement of elements, deformation, changes in characteristics, changes in the leaning angle of buildings, subsidence of supports, ground support for construction elements, and determinaion of force in earth anchors, etc. (Benmokrane et al., 1995; Hayes and Simmonds, 2002; Neild et al., 2005; Sharma et al., 1999).

Wire sensors have a range of benefits. The output signal from a transducer has a frequency character which makes it resistant to external disturbances and the influence of cables transferring data between the transducer and the recording device. This makes it possible to install the transducer in a significant distance away from the location of data acquisition point (Simonetti, 2012). Its simple and solid construction ensures high resistance to mechanical damage and insensitivity to atmospheric conditions. It is also possible to use wireless communication between the transducer and the measuring system (Park et al., 2013).

The most important feature of wire sensors is their high metrological stability, which according to the research conducted by Norwegian Geotechnical Institute - can last for 27 years (DiBiagio, 2003). The features of wire transducers mean that they are still often applied in SHM systems of constructions.

One example of the use of wire measurement techniques on a large scale is the monitoring system of A1 motorway embankment in Poland. This covers an area of approximately 100 thousands $\mathrm{m}^{2}$. Around 15 thousands wire transducers are used there. 
During the last 80 years, the construction and working principle of wire sensors have not changed. They consist of steel wires excited into transverse vibration with the help of an electromagnet (Simonetti, 2012). A change in the force of the wire influences its transverse vibration frequency - and that is the basis for finding the relative extension of the wire, and then defining the deformation of the object to which the sensor has been mounted. The electro-magnet also performs function of a vibration detector.

Wire transducers are designed in principle for measurement of slow-changing or static loads. This limitation results from its working principle, in which successive vibration excitement of the wire is only possible after the previous vibration has subsided. Excessively early excitation may cause mutual interference between vibrations and lead to disturbances and impracticality of the metrological signal. As a result, the maximum excitation frequency of the wire for a typical solution is defined at the level of $1 \mathrm{~Hz}$. As can be seen, the method has limited application in the case of constant measurement with the aim of registering the course of fast-changing changes, e.g. in high objects affected by the wind (Qing et al., 2008), such as chimneys, masts and towers, or machine supports, for example, foundations under turbo-generators, railway and road bridges in the event of para-seismic and seismic disturbance.

In the literature, it is possible to find solutions that allow measurement of quasi-constant fastchanging deformation. In this case, the most important element of the method is the maintenance of natural non-decreasing vibrations of wires by cyclical, in accordance with the phase of wire vibration, excitation with electro-magnetic impulses. This method does not, however, guarantee the continuity of measurement. Some of signals are lost due to the presence of transition states caused by the exciting impulses. There is a high probability that, as a result of violent changes in the measured deformation, the impulse phase will not correlate with the wire vibration phase, which most frequently leads to formation of undesired transition states, and even to suppression of wire vibration.

\section{Maintenance of non-decreasing natural vibration of wires}

One way to excite and maintain non-decreasing natural vibration of wires, according to the authors, is to introduce self-oscillation in wires. Vibrations of this type are well-known in mechanical engineering (Den Hartog, 1956). They appear in non-conservative systems and are capable of independently replenishing lost energy. Their amplitude and frequency are defined by physical parameters of the system. Due to this, they are different from damped vibrations in that they do not fade out in time, and that the frequency of vibrations in the steady state is not dependent on the frequency of external forces.

An example of an equation that describes self-oscillation comes from van der Pol (Atay, 1998)

$$
\frac{d^{2} y}{d t^{2}}-\varepsilon\left(1-y^{2}\right) \frac{d y}{d t}+y=0
$$

This contains a non-linear component $-\varepsilon\left(1-y^{2}\right)(d y / d t)$ responsible for the maintenance of vibrations, which will further be named in this work as the van der Pol component. An example of the solution to (2.1) is presented in Fig. 1. It can be seen that for various initial conditions, the trajectory of the solution winds on a fixed limit cycle. By modifying equation (2.1) to form (2.2), it is possible to provide it with a physical interpretation. This then describes motion of mass $m$ located on a viscoelastic suspension under the force in accordance with the formula of the van der Pol component, see Fig. 2.

$$
m \frac{d^{2} y}{d t^{2}}+b \frac{d y}{d t}+k y=\varepsilon\left(a^{2}-y^{2}\right) \frac{d y}{d t}
$$




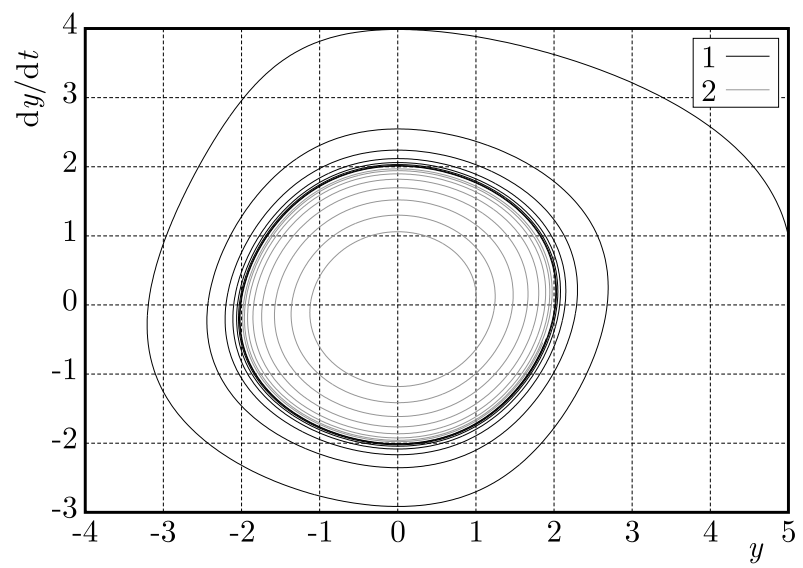

Fig. 1. Phase portrait of Eq. (2.1) for two various initial conditions. Trajectory 1 has a direction field from the external side of the limit cycle contour, while trajectory 2 comes from the internal side

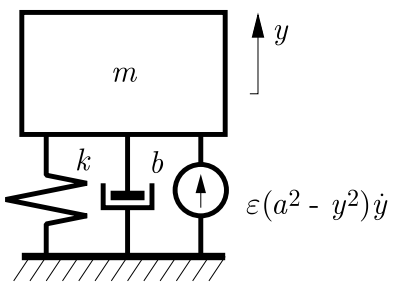

Fig. 2. Model of the mechanical structure representing equation (2.2)

If the absolute value of the coordinate $y$ falls below the value of the parameter $a$, the van der Pol component supplies energy to the system, otherwise it dissipates it. One characteristic feature that the authors of the work have noticed is the lack of turbulent transition states appearing in the event of violent changes in the elasticity coefficient $k$, see Figs. 3a and 3b. Of course, the coordinate $y$ changes its course because it sets a new cycle with a different frequency, however, this process takes place without any significant disturbance to motion. The new vibration frequency of the mass $m$ is set almost immediately.
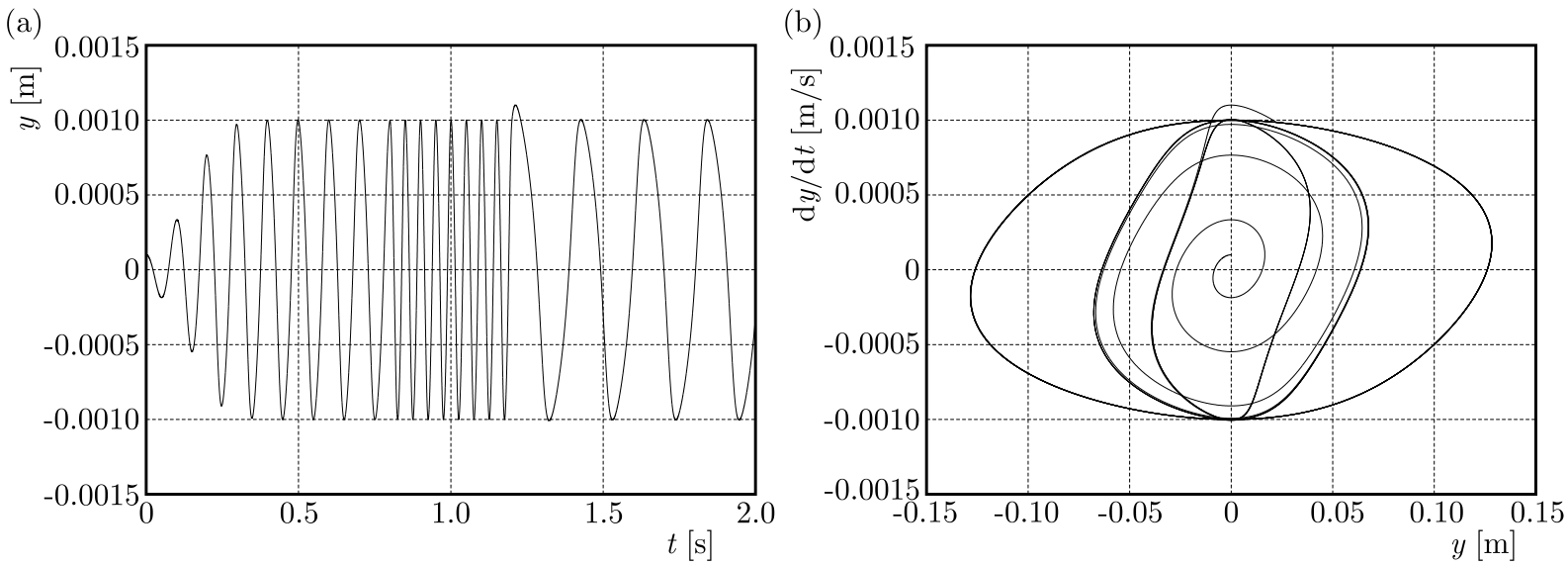

Fig. 3. (a) Course of the coordinate $y$ for stepwise changes of the coefficient $k$ (changes of the coefficient took place at $0.8 \mathrm{~s}$ and $1.2 \mathrm{~s}$ ), (b) trajectory with three limit cycles

This feature of the system has become a basic principle of the application of self-oscillation in wire sensors. In this case, the van der Pol component ensures the maintenance of non-decreasing transverse vibration of the wire, and violent changes in its load (in the longitudinal direction) do not cause any disturbance in its transverse motion. The new vibration frequency is set almost immediately and is ready to be read by a signal processing system. 


\section{Mathematical model of a wire}

Let us analyse a wire simultaneously performing transverse vibration and longitudinal motion, see Fig. 4. Designating by $A$ the field of the wire transverse cross-section, $\rho$ - material density, and $d x$ - the elementary section of the wire, we can write

$$
d m=\rho A d x
$$

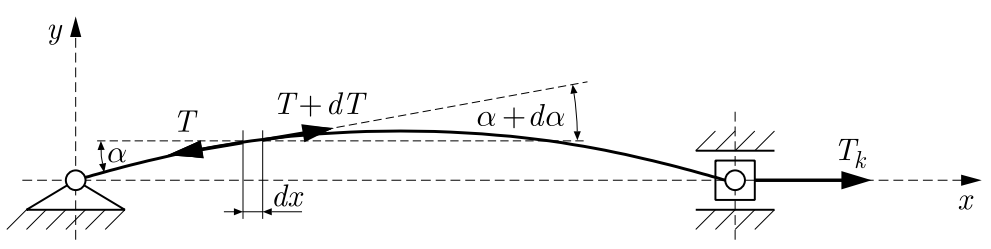

Fig. 4. Model of a wire subjected to longitudinal load

Treating the elementary mass $\mathrm{dm}$ as a material point in planar motion, we can, based on Newton's second law, formulate a dynamic equation of its motion. Designating the location of the mass $d m$ with the coordinates $x$ and $y$, we obtain the equation for transverse motion

$$
d m \frac{\partial^{2} y}{\partial t^{2}}=(T+d T) \sin (\alpha+d \alpha)-T \sin \alpha-b_{y} \frac{\partial y}{\partial t} d x+q(x, t) d x
$$

in which $T$ designates the force in the wire, $b_{y}$ - coefficient of viscous friction calculated as a unit of distance and $q$ - linear density of the external transverse force. Assuming a small value of the angle $\alpha$, equation (3.2) can be simplified to

$$
d m \frac{\partial^{2} y}{\partial t^{2}}=T d \alpha+\alpha d T-b_{y} \frac{\partial y}{\partial t} d x+q(x, t) d x
$$

Expressing $\alpha$ by $\partial y(x, t) / \partial x$ and using dependency (3.1), we can write the equation of the wire transverse motion in form

$$
\rho A \frac{\partial^{2} y(x, t)}{\partial t^{2}}-T(x, t) \frac{\partial^{2} y(x, t)}{\partial x^{2}}+b_{y} \frac{\partial y(x, t)}{\partial t}=\frac{\partial y(x, t)}{\partial x} \frac{\partial T(x, t)}{\partial x}+q(x, t)
$$

In the last equation, the force $T$ is not constant. Its temporary value can be determined based on the equation of motion of the longitudinal mass $d m$, where $u$ designates the displacement of the elementary section of the wire along its axis

$$
d m \frac{\partial^{2} u}{\partial t^{2}}=-T \cos \alpha+(T+d T) \cos (\alpha+d \alpha)-b_{x} \frac{\partial u}{\partial t} d x
$$

By using a small-angle approximation as well as assuming uniformity of the material, we obtain the equation of wire longitudinal motion

$$
\frac{\partial^{2} u(x, t)}{\partial t^{2}}+\frac{b_{x}}{A \rho} \frac{\partial u(x, t)}{\partial t}-\frac{E}{\rho} \frac{\partial^{2} u(x, t)}{\partial x^{2}}=0
$$

where $E$ is Young's modulus, $b_{x}$ - coefficient of viscous friction calculated as a unit of distance.

Based on Hook's law, we can express the force in the wire by its local deformation, that is

$$
T=A \sigma=A E \varepsilon=A E \frac{\partial u}{\partial x}
$$


or

$$
\frac{\partial T}{\partial x}=E A \frac{\partial^{2} u}{\partial x^{2}}
$$

By placing dependency (3.8) into equation (3.4), we obtain

$$
\rho A \frac{\partial^{2} y(x, t)}{\partial t^{2}}+b_{y} \frac{\partial y(x, t)}{\partial t}-A E \frac{\partial u(x, t)}{\partial x} \frac{\partial^{2} y(x, t)}{\partial x^{2}}=E A \frac{\partial y(x, t)}{\partial x} \frac{\partial^{2} u(x, t)}{\partial x^{2}}+q(x, t)
$$

The set of equations (3.6) and (3.9) represent the basis for a mathematical description of wire motion, whose points perform transverse and longitudinal movement coupled with each other.

The method for fixing the wire is defined by the boundary conditions. In the considered cases, the wire is mounted unilaterally. The second end is conducted linearly and is affected by the external force $T_{k}$. On this basis, it is possible to write the following conditions

$$
y(0, t)=0 \quad y(l, t)=0
$$

and

$$
u(0, t)=0 \quad \frac{\partial u(l, t)}{\partial x}=\frac{1}{E A} T_{k}
$$

\section{Adaptation of van der Pol's equation to maintain non-decreasing natural vibrations of a wire}

Transferring the idea of applying self-oscillation to wire gauges is presented in Fig. 5. Untied between two points, it is propelled to move transversely using the Lorentz force created as a result of the interference of current i flowing through the wire as well as the magnetic field created by fixed magnets. The intensity of current $\mathrm{i}$ is shaped by the electric system working in a feedback loop, in which the input signal is the voltage induced in the magneto-electric transducer. In order to adapt the input voltage to an electronic system, it is first amplified, and then undergoes integration in time so that the wire displacement signal is obtained from the amplitude transducer. After transformation of the signal by the multiplier-summing systems realizing the van der $\mathrm{Pol}$ component, it is amplified to a value that allows creation of a force ensuring vibrations at a level which enable their recording.

\section{Results of computer simulations}

Based on the equations from the previous Section, computer simulations of motion of the wire subjected to a stepwise load have been performed. In order to solve the differential equation of motion, the finite difference method with the "time step" variant has been applied. After replacing the partial derivatives in equations (3.6) and (3.9) with the following finite differences

$$
\begin{array}{ll}
\frac{\partial y}{\partial x}=\frac{y(i+1, j)-y(i, j)}{\Delta x} & \frac{\partial^{2} y}{\partial x^{2}}=\frac{y(i+1, j)-2 y(i, j)+y(i-1, j)}{(\Delta x)^{2}} \\
\frac{\partial y}{\partial t}=\frac{y(i, j+1)-y(i, j)}{\Delta t} & \frac{\partial^{2} y}{\partial t^{2}}=\frac{y(i, j+1)-2 y(i, j)+y(i, j-1)}{(\Delta t)^{2}}
\end{array}
$$

and

$$
\begin{array}{ll}
\frac{\partial u}{\partial x}=\frac{u(i+1, j)-u(i, j)}{\Delta x} & \frac{\partial^{2} u}{\partial x^{2}}=\frac{u(i+1, j)-2 u(i, j)+u(i-1, j)}{(\Delta x)^{2}} \\
\frac{\partial u}{\partial t}=\frac{u(i, j+1)-u(i, j)}{\Delta t} & \frac{\partial^{2} u}{\partial t^{2}}=\frac{u(i, j+1)-2 u(i, j)+u(i, j-1)}{(\Delta t)^{2}}
\end{array}
$$




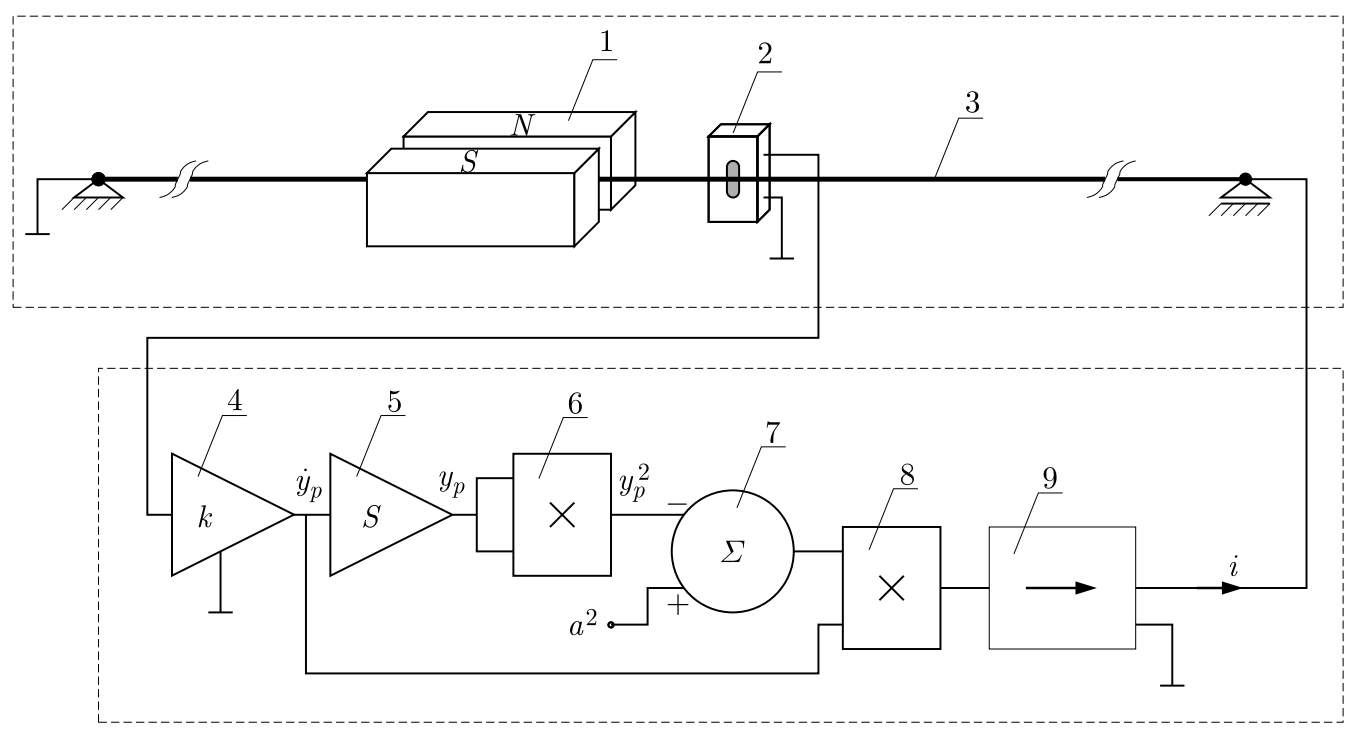

Fig. 5. Sketch of the control system of the wire gauge based on the van der Pol equation. 1 - fixed magnet, 2 - magneto-electric transducer, 3 - wire, 4 - voltage amplifier, 5 - integrating system, 6,8 - multiplying system, 7 - summing system, 9 - current amplifier

we obtain relationships for the transverse displacement $y(i, j+1)$ and longitudinal displacement $u(i, j+1)$ at points of the wire with reference to the displacement from the previous time moment, that is, $y(i, j), u(i, j)$

$$
\begin{aligned}
& y(i, j+1)=\left\{\frac{q(i, j)}{\rho A}-\frac{1}{(\Delta t)^{2}}[y(i, j-1)-2 y(i, j)]\right. \\
& \left.+\frac{u(i+1, j)-u(i, j)}{(\Delta x)^{3}} \frac{E}{\rho}[y(i+1, j)-2 y(i, j)+y(i-1, j)]+\frac{b_{y}}{\rho A} \frac{1}{\Delta t y(i, j)}\right\} \\
& \cdot\left[\frac{1}{(\Delta t)^{2}}+\frac{b_{y}}{\rho A(\Delta t)}\right]^{-1} \\
& u(i, j+1)=\left\{-\frac{1}{(\Delta t)^{2}}[u(i, j-i)-2 u(i, j)]+\frac{E}{\rho} \frac{1}{(\Delta x)^{2}}[u(i+1, j)-2 u(i, j)+u(i-1, j)]\right. \\
& \left.+\frac{b_{x}}{\rho A(\Delta t)} u(i, j)\right\}\left[\frac{1}{(\Delta t)^{2}}+\frac{b_{x}}{\rho A(\Delta t)}\right]^{-1}
\end{aligned}
$$

where

$$
q(i, j)=\varepsilon\left[a^{2}-y(i, j)^{2}\right] \frac{y(i, j+1)-y(i, j)}{\Delta t}
$$

describes the action of the van der Pol component.

For simulation purposes, the following parameters have been adopted:

$$
\begin{aligned}
& l=0.15 \mathrm{~m} \\
& D=0.0002 \mathrm{~m} \\
& \rho=7800 \mathrm{~kg} / \mathrm{m}^{3} \\
& T_{k}=14.115 \mathrm{~N} \\
& \tau=24.5 \mathrm{E}-05 \mathrm{~kg} / \mathrm{m} \\
& E=220 \mathrm{GPa} \\
& a=0.0001 \mathrm{~m}
\end{aligned}
$$

- length of the wire,

- diameter of the wire,

- density of the wire,

- tension at the end of the wire,

- linear density of the wire,

- Young's modulus,

- parameter of the van der Pol component.

Figure $6 \mathrm{a}$ presents the longitudinal displacement $\mathrm{u}$ of the point lying in the middle of the wire, when the changes in the load took place at the moments $t_{1}=0.009 \mathrm{~s}$ with the value 
$T_{k}=14.12 \mathrm{~N}$ at $T_{k}=7.06 \mathrm{~N}$ as well as $t_{2}=0.0175 \mathrm{~s}$ with the value $T_{k}=7.06 \mathrm{~N}$ at $T_{k}=28.23 \mathrm{~N}$. Figure $6 \mathrm{~b}$ presents the respective changes of transverse vibration of the same point starting from the initial state representing the equilibrium position until the development of set vibrations. The vibration amplitude, in accordance with theoretical knowledge, obtains a value twice as high as the value of the parameter $a$, in other words, $0.2 \mathrm{~mm}$. The vibration frequency of the first form in this section equals $800 \mathrm{~Hz}$, and in the next two time sections it equals $562 \mathrm{~Hz}$ and $1135 \mathrm{~Hz}$, respectively.

(a)

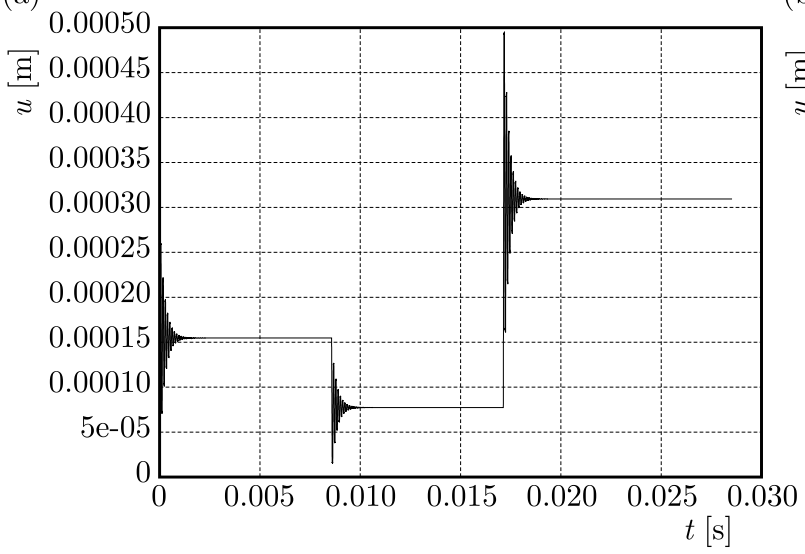

(b)

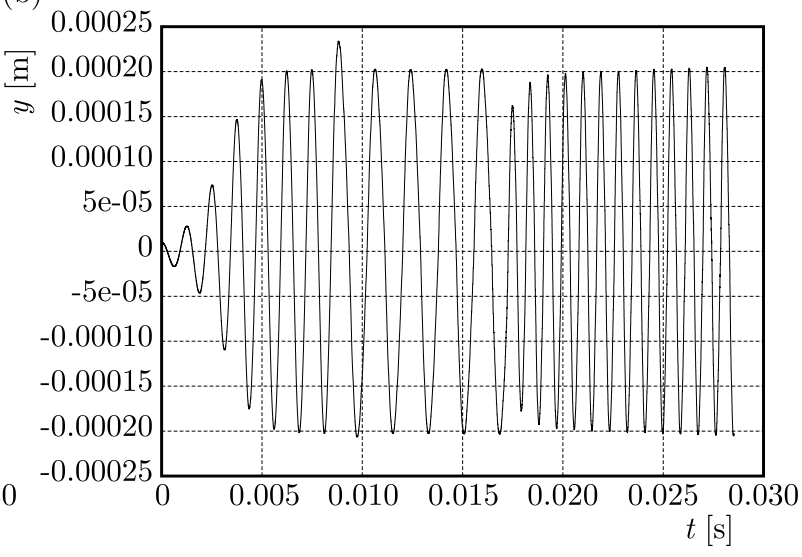

Fig. 6. (a) Course of the longitudinal displacement $u$ at the point lying in the middle of the wire $(x=l / 2)$; (b) course of the transverse displacement of the point lying in the middle of the wire $(x=l / 2)$

The frequency values differ from the values obtained based on the below given theoretical formula (5.5) for free wires by about $8 \mathrm{~Hz}(1 \%)$, which is reflected in the scientific literature

$$
f_{0}=\frac{1}{2 l} \sqrt{\frac{T_{k}}{\tau}}
$$

where $f_{0}$ is the natural vibration frequency of the wire.

As in the case of a discrete system, transfer between new work states takes place without causing turbulent transition states in the transverse direction, and the new frequencies are set almost immediately. There is a noticeable lack of any significant influence of the transition states appearing along the wire.

\section{Modification of the van der Pol component}

The regulator shaping the van der Pol force component requires the use of a displacement signal of the vibrating mass. From the magneto-electric transducer registering wire motion, we obtain, however, a signal proportional to velocity of the mass motion. Although transforming the velocity signal to a displacement signal is theoretically easy, in practice we encounter the problem of constant value, trend and filtering process leading to phase shift. There is, however, a simpler solution proposed by J. Michalczyk ${ }^{1}$. Namely, instead of using the displacement signal in the van der Pol component, it is possible to use a vibration velocity signal provided directly by the magneto-electric transducer. Equation (2.2) then takes form of the following equation

$$
m \frac{d^{2} y}{d t^{2}}+b \frac{d y}{d t}+k y=\varepsilon\left[a^{2}-\left(\frac{d y}{d t}\right)^{2}\right] \frac{d y}{d t}
$$

\footnotetext{
${ }^{1}$ Faculty seminar. Faculty of Mechanics and Vibroacoustics AGH.
} 
The solution to equation (6.1) for $a=0.04$ is presented in Figs. $7 \mathrm{a}$ and $7 \mathrm{~b}$ in form of time and phase portraits, respectively. As can be seen, the equation shows all the features of the equation describing self-oscillation, including the existence of a limit cycle on which trajectories of the phase solution have a direction field from the inside or the outside. And just as in the case of the initial equation, stepwise changes in the coefficient of elasticity $k$ do not lead to undesired transition states in the solution of the equation. This fact becomes a basis for removal of integrating system " 5 " from the scheme presented in Fig. 5.
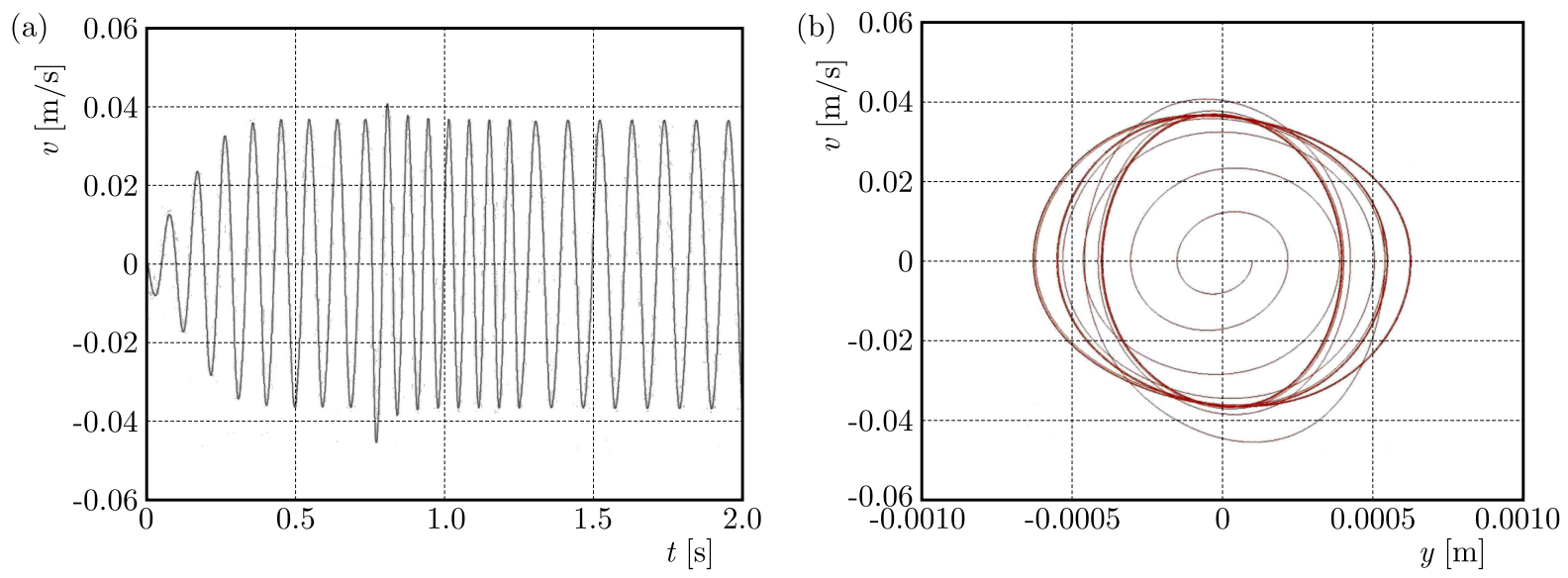

Fig. 7. Courses of the solution to equation (6.1) for three stepwise variables of rthe parameter $k$ : (a) time form, (b) phase portrait

\section{Construction of the test stand}

Based on the scheme in Fig. 5, a physical model of the system has been constructed. It consists of a mechanical part, including the wire system together with transducers, as well as an electrical part which performs the task of maintaining self-excited wire vibrations.

The electrical part is presented in Fig. 8. The signal from the magneto-electric transducer after initial amplification in the U1 voltage amplifier (Fig. 8b) is conducted to the pins of two integrated systems, U2 and U3 (Fig. 8a). These are AD633JN/A summing-multiplying systems, using which the feedback loop signal is obtained. System U2 formulates the signal $a^{2}-(d y / d t)^{2}$ in which the value of the parameter $a$ is set with the help of potentiometer Pt1. In turn, system U3 formulates the signal of the sum of the output signals of system U2 (end 7) and the signal of vibration velocity $d y / d t$ - input 3 of $\mathrm{U} 3$ system. Such an obtained signal is then amplified with an current amplifier. In the photograph presented in Fig. 9, the elements of the mechanical part of the system are shown. A uniform guitar string fastened between two pegs (Fig. 9a) is used as a wire. The pegs are mounted through the bars to a steel base, which simultaneously performs function of the deformed element. In the central part of the wire, two flat neodymium magnets (Fig. 9c), separated from each other with isolation pads, are mounted. The pads have the task of setting the suitable depth of the air passage, which should ensure free vibration of the wire and guarantee interaction between the magnets and the wire at a level sufficient to develop vibration at the measurement amplitude. A guitar magneto-electric transducer is located in the neighbourhood of the magnets at a distance ensuring the lack of significant interference of the magnetic field (Fig. 9b).

Such a workstation enables research to be carried out into wire vibration formed as a result of changes in its tension and interference of the Lorentz forces formulated in the non-linear van der Pol component designated for generation of self-oscillation. 

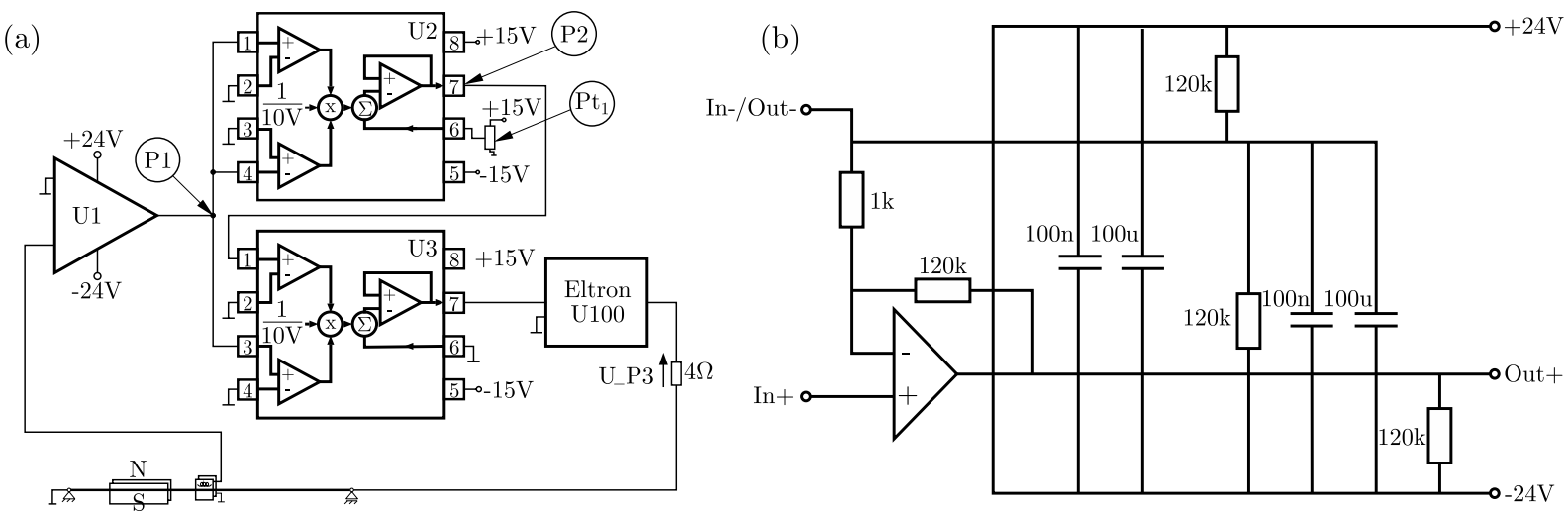

Fig. 8. (a) U2, U3: AD633JN/A multiplying systems, (b) input amplifier U1

(a)

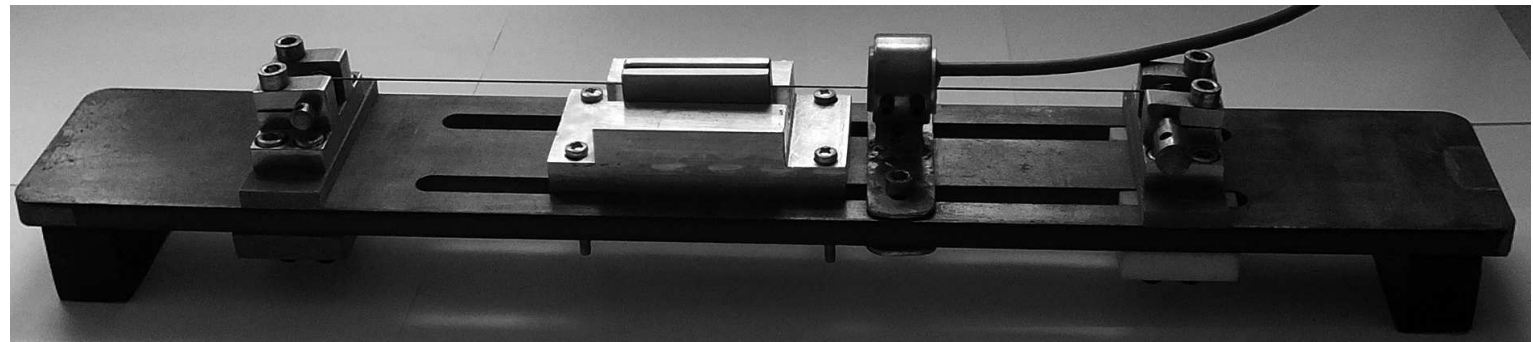

(b)

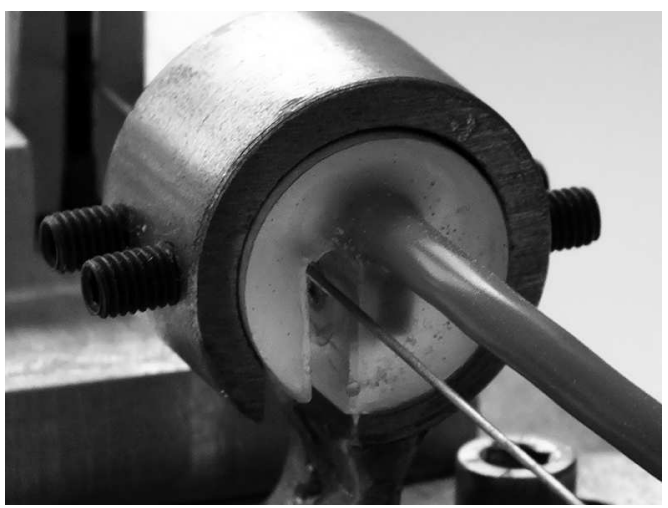

(c)

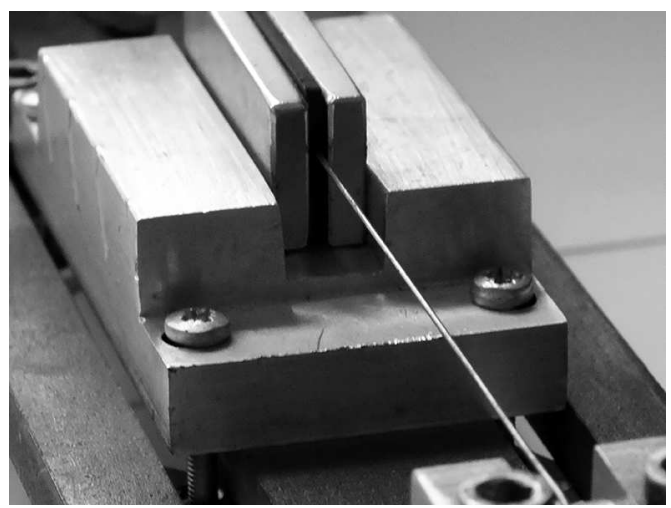

Fig. 9. Photographs of the wire transducer: (a) wire mounted to the deformed base, (b) magneto-electric transducer, (c) neodymium fixed magnets

\section{Results of laboratory tests}

Measurements have been carried out with a two-channel Hewlett Packard HP3560A recorder. This device allows recording of short signals, tens of milliseconds long, at a sampling frequency equalling $2.5 \mathrm{kHz}$. Therefore, in the measurements presented below, only set states have been captured (transient states on the base of observation did not develop noticeably). Figure 10a presents trajectories recorded at two points of the system, that is, the output of U1 input amplifier (course designated as P1) as well as the output of U2 multiplier system (course designated as P2). Figure 10b presents the course recorded at P1 as well as the course of voltage in the output resistor U_P3 of the current amplifier system. Both figures present vibration for an initially tensioned wire, which in the second case slightly changes the parameter $a$.

The courses presented in Fig. 11a refer to the case in which the wire is significantly tensioned. As in the previous cases, it presents courses of the output from the input amplifier and the multiplying system. The vibration frequency of the wire increased from $800 \mathrm{~Hz}$ to $2200 \mathrm{~Hz}$. 
(a)

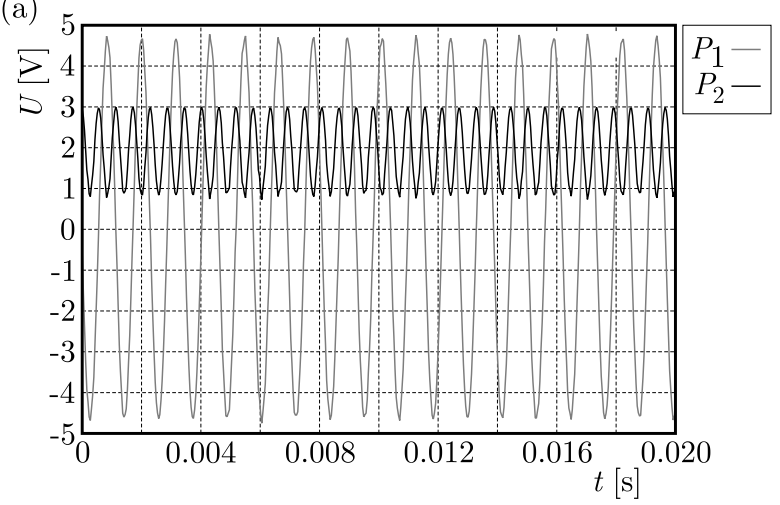

(b)

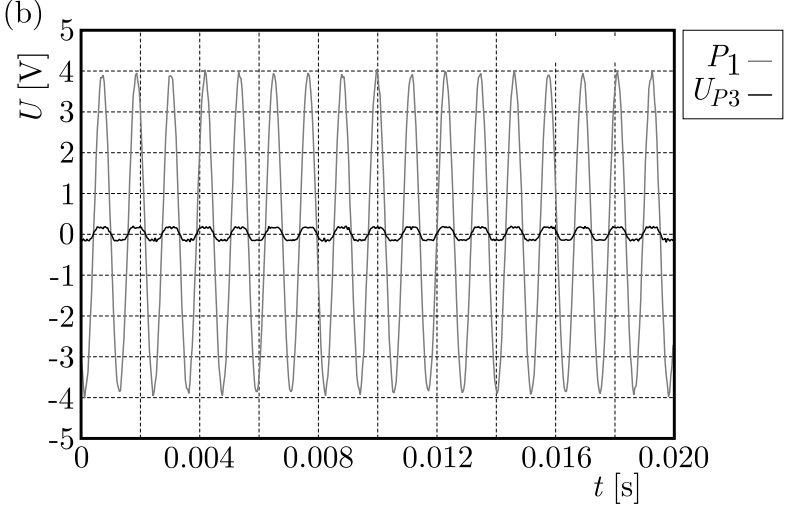

Fig. 10. Wire under initial load: (a) course of voltage signals at points P1 and P2 of the system presented in Fig. 8, (b) voltage in the output of U1 amplifier (P1) and in output resistor of the current amplifier (U_P3)

The final figure, Fig. 11b, presents the course after removing the load and after significantly reducing the value of the parameter $a$.

(a)

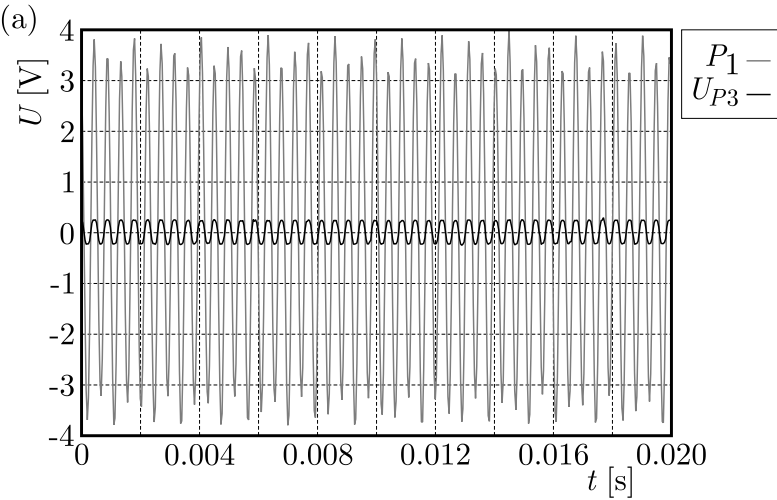

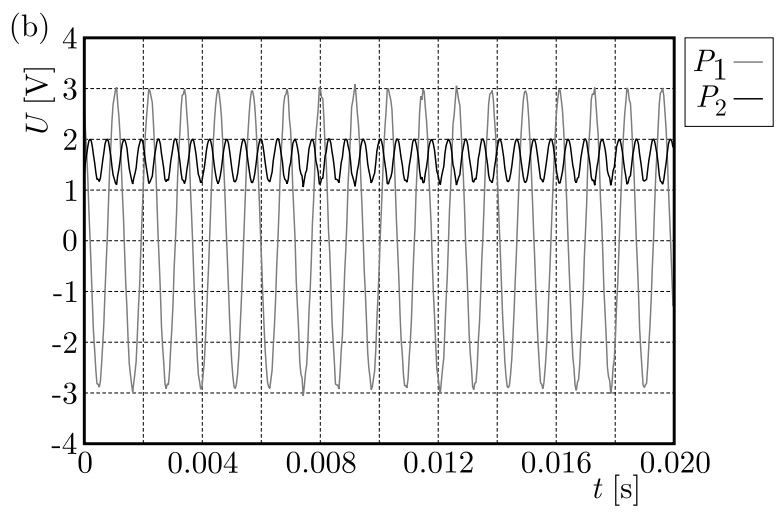

Fig. 11. (a) Wire under load: (a) voltage course in the output of U1 amplifier (P1) and in output resistor of the current amplifier (U_P3), (b) wire after removal of the load. Courses of voltage signals at points P1 and P2

While comparing the figures, it is possible to state that the task of maintaining non-decreasing self-oscillation in the wire, regardless of the wire tension, has been achieved, and the vibration velocity amplitude can be set by changing the value of one of the regulator parameters. Based on the observations of the wire behaviour under violent changes of the load, the formation of transition states which would lead to motion of the wire with chaotic vibrations and the loss of measurement ability, has not been observed.

\section{Summary}

The aim of the presented research is to show the possibility of using self-oscillation to maintain non-decreasing natural vibrations in a wire resistant to violent changes in the tension.

On the basis of the presented results, it can be stated that this idea has been confirmed in simulation tests and then in real conditions on the laboratory stand. Self-oscillation does not show a clear tendency to develop turbulent transition states during violent changes in the load. In fact, the opposite case has been observed, the transition from one load state to the second one took place very smoothly, without the loss of any period. 
The presented solution may be used to construct wire sensors with special purposes, resistant to impact or fast-changing loads. The construction of the system significantly differs from the one universally used in measurement techniques with single-coil wire sensors, in which the coil itself performs function of both input sensor tracing motion of the wire and vibration exciter. This solution is more expensive and energy-consuming. However, as opposed to conventional solutions, it does not bring the risk of losing synchronization between the system stimulating and recording the vibrations.

\section{Acknowledgements}

The study described in this paper has been executed within the project No. 11.11.130.955

\section{References}

1. Atay F.M., 1998, Van der Pol's oscillator under delayed feedback, Journal of Sound and Vibration, 218, 2, 333-339

2. Barr P.J., Petroff S.M., Hodson D.J., Thurgood T.P., Halling M.W., 2012, Baseline testing and long-term monitoring of the Lambert Road Bridge for the long-term bridge performance program, Journal of Civil Structural Health Monitoring, 2, 2

3. Benmokrane B., Chekired M., Xu, H., 1995, Monitoring behavior of grouted anchors using vibrating-wire gauges, Journal of Geotechnical Engineering, 121, 6, 466-475

4. Bordes J.-L., 2011, Dams and large scale experiments: monitoring and control. Documents pour l'historie des technigues, no. 20, semestre 2011

5. Den Hartog J.P., 1956, Mechanical Vibrations, 4th Edition, McGraw-Hill Book Company, New York

6. DiBiagio E., 2003, A case study of vibrating-wire sensors that have vibrated continuously for 27 years, 6th International Symposium on Field Measurements in Geomechanics (FMGM), Oslo, Norway

7. Gastineau A., Johnson T., Schultz A., 2009, Bridge Health Monitoring and Inspections A Survey of Methods Department of Civil Engineering University of Minnesota. Final Report No. $\mathrm{MN} / \mathrm{RC} 2009-29$

8. Hayes J., Simmonds T., 2002, Interpreting strain measurements from load tests in bored piles, Ninth International Conference On Piling And Deep Foundations, Nice, 663-669

9. Neild S.A., Williams M.S., McFadden P.D., 2005, Development of a vibrating wire strain gauge for measuring small strains in concrete beams, Strain, 41, 1, 3-9

10. Park H.S., Lee H.Y., Choi S.W., Kim Y., 2013, A practical monitoring system for the structural safety of mega-trusses using wireless vibrating wire strain gauges, Multidisciplinary Digital Publishing Institute, Sensors, ISSN:1424-8220

11. Qing N.Y., Yong X., YAng L.W., Peng Z., 2008, Development of a structural health monitoring system for guangzhou new TV tower, Advances in Science and Technology, 56, 414-419

12. Sharma V.M., NAIR M.D., Meller S., 1999, The use of vibrating wire strain gauges in geotechnics, International Conference on Offshore and Nearshore Geotechnical Engineering, GEOShore

13. Simonetti A., 2012, A Measurement Technique for the Vibrating Wire Sensors, NORCHIP, Copenhagen, Denmark

14. Wenzel H., 2009, Health Monitoring of Bridges, John Wiley \& Sons, Ltd. 